\title{
On the locus of the stimulus suffix effect
}

\author{
JAMES S. NAIRNE \\ University of Texas, Arlington, Texas 76019 \\ and \\ ROBERT G. CROWDER \\ Yale University, New Haven, Connecticut 06520
}

\begin{abstract}
Two experiments contrasted short-term memory and sensory memory (precategorical acoustic storage) explanations of the stimulus suffix effect. Experiment 1 attempted to interfere with recency recall by forcing subjects to encode nonverbal acoustic suffixes into a verbal, articulatory code. Subjects in this condition showed recall that was comparable to that of subjects who were told to ignore the suffixes. However, Experiment 2 showed that silently mouthed articulation of a suffix can produce interference on recall of recency items, although not as much as that produced by vocal articulation. The results are discussed in terms of current models of the stimulus suffix effect.
\end{abstract}

For immediate recall of lists presented vocally, correct performance depends partly upon whether an extra word, or suffix, is attached to the end of the memory list. Serial recall of lists containing such a stimulus suffix is sharply impaired for the last few serial positions relative to recall of control lists for which there is no added suffix (Crowder, 1967; Dallett, 1965). This result, termed the stimulus suffix effect, depends upon the physical similarity between the suffix and list items and not upon their conceptual similarity. For example, changing the speaker's voice quality or the spatial location of the suffix reduces the effect, whereas varying the semantic similarity between the suffix and list items has no effect (Morton, Crowder, \& Prussin, 1971).

Crowder and Morton (1969) suggested that the suffix effect could be interpreted in terms of masking within precategorical acoustic storage, a sensory memory store capable of holding a small amount of auditory information in a relatively raw, uncategorized format. Although easily disrupted by subsequent auditory input, the contents of precategorical acoustic storage remain available for limited amounts of time to aid concurrent memory processing, especially during the interval between the end of the list presentation and the beginning of written recall. During this period, the subject presumably checks his short-term memory code for the end of the presented list with activation in the auditory store. The recall process itself is then based only on the short-term memory code. Due to its limited capacity, precategorical acoustic

This research was conducted while James Nairne was at Yale University and was supported by Grant BNS 8005838 from the National Science Foundation. The authors wish to express their gratitude to Virginia Walters for her considerable assistance in all stages of this project. Requests for reprints can be addressed to James Nairne, Department of Psychology, University of Texas, Arlington, Texas 76019. storage is thought to be particularly beneficial to the last memory item and, thus, to play some role in producing the standard recency advantage found in immediate recall of acoustically presented items. However, with the addition of a stimulus suffix, provided that the suffix is acoustic and physically similar to the preceding list items, the contents of precategorical acoustic storage are disrupted, and recall of the last item on the list is impaired.

Although the precategorical acoustic storage model has met with a reasonable level of acceptance, a number of alternative explanations of the suffix effect have been proposed (see Crowder, 1978, for a review). Of special interest is a recent account by Spoehr and Corin (1978), who investigated an earlier suggestion by Massaro (1972) that the suffix effect might be more easily interpreted as interference in short-term memory rather than as a manifestation of an auditory sensory memory system. Spoehr and Corin (1978) placed the locus of the suffix effect at the point at which the subject recodes list information into a form suitable for immediate recall. This recoding presumably occurs as a short-term memory process, and the end result is seen as some kind of articulatory code (Hintzman, 1967). Therefore, in accordance with established short-term memory principles, any item that is in the process of being coded might be interfered with by a suffix that is also in articulatory form.

Obviously, this account places importance upon the similarity between the suffix and list items. It differs from the precategorical acoustic storage account, however, in that this similarity is not expressed in an absolute spectral or acoustic sense (Crowder, 1978) but, rather, as a relationship between the suffix and list items as coded into the verbal memory system. The damaging effect of the suffix is not, therefore, a unique function of its acoustic properties but, instead, follows from 
general similarities in the coding format between vocally presented suffix and list items. Unlike the precategorical acoustic storage account, this recoding hypothesis predicts that it is possible, under certain conditions, to produce a suffix-like effect using a suffix that is not acoustic.

To provide some evidence for this last point, Spoehr and Corin (1978) conducted an experiment in which the visual, articulatory, and acoustic properties of a digit suffix were systematically manipulated. All subjects listened to lists of eight digits presented vocally by the experimenter, who was visible to the subjects at all times. The lists ended with the experimenter (1) speaking aloud an additional digit suffix, (2) silently articulating, or mouthing, a digit suffix, (3) showing a suffix written on a card, or (4) doing nothing. Subsequent comparisons of the spoken suffix condition and the visual and nosuffix conditions revealed the standard suffix effect: The spoken suffix clearly produced interference relative to the other two conditions. The most important finding was that the mouthed suffix condition, despite the lack of any auditory information, showed a pattern of recall similar to that of the spoken suffix condition. Spoehr and Corin reasoned that since the subject was probably using an articulatory code in short-term memory for the list itself, the salient articulatory features produced by watching a mouthed suffix were sufficient to produce substantial interference in the coding of the last few list items occurring immediately prior to recall. Clearly, this finding is inconsistent with the original precategorical acoustic storage account, which specifies that auditory input is a necessary requirement for the production of suffix interference.

A recent series of experiments by Campbell and Dodd (1980) has provided further evidence consistent with a short-term memory locus explanation and inconsistent with the precategorical acoustic storage account. Campbell and Dodd demonstrated that an auditory suffix, when placed at the end of a silently articulated, lipread list, produced substantial interference in the recency portion of immediate free recall when compared with a no-suffix condition. They concluded that lip-read speech is encoded into a format that shares properties with the encoding format of heard speech, and it is this common form of coding that is responsible for the interference effect.

The present article describes the results of two experiments that were designed to test further implications of general short-term memory accounts of the stimulus suffix effect. Experiment 1 attempted to produce a suffix effect with a nonverbal acoustic suffix by forcing subjects to encode the suffix into a verbal, articulatory code. Experiment 2 attempted to replicate Experiment 1 and to provide more evidence of the role of silent articulation in producing a suffix effect. Both experiments compared nonacoustic and nonverbal suffix manipulations with standard acoustic suffix controls.

\section{EXPERIMENT 1}

The purpose of the first experiment was to induce subjects to encode a nonverbal acoustic suffix (one that would not ordinarily produce a suffix effect) into a form suitable for immediate written recall. The idea was that the innocuous, nonverbal suffix might become a potent suffix if subjects transformed it into a verbal articulatory form, according to the Spoehr-Corin (1978) reasoning. Each subject listened to 60 lists of nine digits that ended with (1) the word "high," (2) the word "low," (3) a high tone, (4) a low tone, or (5) no sound at all. One group of subjects, the "ignore" group, was told that the suffixes were of no particular importance in the experiment other than as signals for the beginning of the recall period. In contrast, a second group of subjects, the "attend" group, was told to pay close attention to the suffixes when they occurred. Each subject in the attend group was instructed to begin recall of the digit list by writing the letter " $\mathrm{H}$ " if the suffix was the word "high" or a high $(1,000-\mathrm{Hz})$ tone and the letter " $\mathrm{L}$ " if the suffix was the word "low" or a low $(200-\mathrm{Hz})$ tone. This modification of the standard suffix procedure was intended to guarantee subjects' attention to the suffix item, a modification first used by Morton and Holloway (1970).

If the locus of the stimulus suffix effect is in the short-term memory recoding stage, during which the subject processes stimulus information into a form appropriate for recall, then the amount of interference produced by the suffix should depend upon how that suffix is coded relative to the remaining items in the list (Spoehr \& Corin, 1978). For lists of verbal items, a suffix should interfere to the extent that it contains, or is recoded into, verbal features. This means that for both attend and ignore subjects, the words "high" and "low" should be effective suffixes because, as in the standard suffix demonstration, they are presented in the same format as the memory items. The between-group comparison of most interest, then, centers upon recall of the lists ending with tones. For the attend group, the tones have to be given verbal labels (" $H$ " or " $L$ ") and processed for immediate written recall. Thus, this recoding process should produce significant interference in the recency portion of the recall curve relative to the nosuffix condition, according to the hypothesis that shortterm memory is the locus of the suffix effect. In fact, there would be little reason to expect the tone and word suffix conditions to differ significantly in this group, because both suffixes must be coded into the same final format. On the other hand, for the ignore group, the tones should be ineffective suffixes because they neither contain nor are recoded into verbal features.

In contrast to these predictions, the precategorical acoustic storage account predicts no interaction between groups. This is because the interfering effect of the suffix is due to its physical, acoustic similarity to the list 
rather than to its final coding format. Therefore, only the word suffixes should interfere selectively with the recency portion of the immediate recall curve.

\section{Method}

Subjects. Subjects were 24 young adults from the Yale University summer community. Each was paid $\$ 3$ for participating in a single experimental session that lasted approximately $1 \mathrm{~h}$.

Materials and Design. Each of the 60 stimulus trials was a random permutation of the digits 1-9. One recorded version of each of the nine numerals was digitized and stored in the Haskins Laboratories pulse code modulation system. These tokens, each $500 \mathrm{msec}$ long, were then used with automatic sequencing and timing programs to produce the experimental tapes. Thus, the stimuli sounded identical throughout the experiment. Each trial began with the word "Ready," spoken in the same male voice as the recorded stimuli, followed after $1 \mathrm{sec}$ by the nine digits separated by an interstimulus interval of $200 \mathrm{msec}$. The suffixes, which occurred with the same timing parameters, were the words "high" and "low" and a high and a low tone $(1,000$ and $200 \mathrm{~Hz}$, respectively). Fifteen seconds were allowed for recall between trials.

The design was between groups with respect to the attention variable: One independent group (the attend group) received instructions to attend to and report the specific suffixes that occurred, and a second independent group (the ignore group) was told to ignore the suffixes. The particular suffix received was a within-subjects variable. The 60 lists were divided into three blocks of 20 trials: One block contained a random ordering of the suffixes "high" and "low," one block contained a random ordering of the high and low tones, and one block contained no suffixes. The order in which a particular block occurred, as well as the type of suffix that ended the list, was completely counterbalanced across subjects.

Procedure. All subjects were instructed that the purpose of the experiment was to study how people remember long numbers. They were asked to listen to each tape-recorded list and then, as soon as all nine digits had been spoken, to write them in order, from left to right, without going back to correct mistakes. Immediately prior to the beginning of the first trial block, all subjects received a short pretest that consisted of the presentation of the different suffixes in a variety of orders. The suffixes were presented to insure that subjects could distinguish between the high and low tones. No specific instructions were given for the pretest; the suffixes were introduced as signals that would occur at the end of two of the three 20-trial blocks.

Each subject in the attend group $(\mathrm{N}=12)$ was told that in addition to attending to the digit series, he was to pay particular attention to the suffixes when they occurred. Depending upon the specific suffix block, subjects were instructed to indicate the suffix in a space to the left of the trial number on their answer sheets. Thus, the identification of the suffix was the first thing the subject did during the recall period. For the suffix block containing the words "high" and "low," subjects wrote an " $H$ " if the suffix was the word "high" and an " $L$ " if the suffix was the word "low." Similarly, for the tone suffix block, subjects wrote an " $H$ " if the suffix was a high tone and an " $L$ " if the suffix was a low tone. For the no-suffix block, subjects were instructed to place a check mark or dash in the appropriate space. Although subjects were free to choose the type of mark they used, they were asked to remain consistent throughout the trial block.

Subjects in the ignore group $(N=12)$ were told that the suffixes were signals for the end of each list and of no particular importance in the experiment. Subjects were instructed to begin recall, however, by placing a check mark or dash in the space to the left of each trial number. This requirement was included to make the subjects' overall recall behavior more comparable to that of subjects in the attend group. In this case, the subjects were asked to keep the mark consistent throughout the trial types.

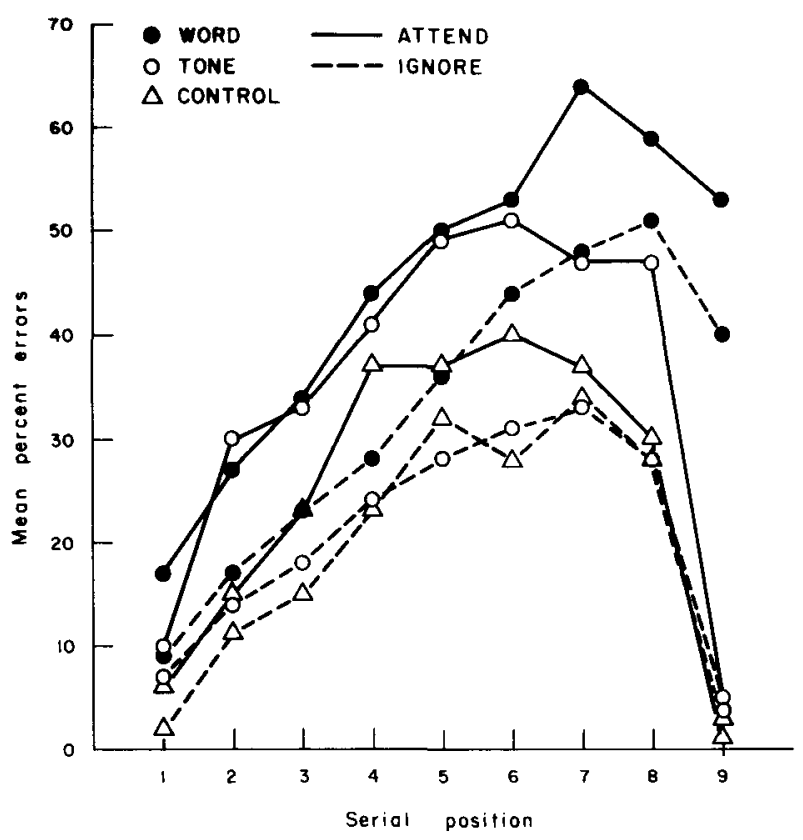

Figure 1. Mean error data for the attend and ignore groups in Experiment 1. The results are plotted separately for each group as a function of serial position.

\section{Results}

Subjects in the attend group were able to discriminate between the different suffixes when they occurred. Only two errors were made in the entire experiment for recall of the presented suffix (i.e., "H" or "L").

The results of principal interest are displayed in Figure 1, which shows the mean percentage of recall errors for each group as a function of serial position. An error occurred if an item was not recalled or if an item appeared in the incorrect serial position. The results are plotted separately for each of the suffix types (words, tones, and no-suffix control). As Figure 1 clearly shows, there was a highly reliable effect of serial position $[F(8,176)=38.36, \mathrm{MSe}=11.89, \mathrm{p}<.01]$, suffix type $[\mathrm{F}(2,44)=32.22, \mathrm{MSe}=18.75, \mathrm{p}<.01]$, and interaction of serial position with suffix type $[F(16,352)=$ $14.3, \mathrm{MSe}=3.8, \mathrm{p}<.01]$. However, the main effect of the between-group manipulation (attend vs. ignore) was not significant $[\mathrm{F}(1,22)=3.06, \mathrm{MSe}=236.04, \mathrm{p}>.05]$, nor did it interact significantly with any other variable in the experiment. Thus, the apparent differences between the attend and ignore groups in the control condition, on the interior serial positions, represent nonsignificant sampling variability in subjects.

\section{Discussion}

The results obtained in Experiment 1 replicated quite nicely the general recall pattern found in other experiments of this type (Morton et al., 1971). The main effect of serial position was representative of good performance for the primacy and recency portions of the list; this result is characteristic of immediate serial recall. Moreover, the advantage in recency recall was reduced by the addition of a word suffix, relative to recall of lists 
with a physically disparate suffix (a tone) or to a nosuffix condition; this is the standard suffix effect.

However, the important feature of this experiment was the comparison of the recall patterns for the attend and ignore groups. In contrast to the predictions of a short-term memory recoding hypothesis, correct performance on the last item in the lists that were followed by a tone suffix was not affected in the attend group relative to the ignore group. Since subjects in the attend group were required to process the word and tone suffixes into the same final format (the letter " $H$ " or "L"), a comparable pattern of interference should have been observed for both these suffix conditions. Instead, recency recall was dramatically affected only when the word suffixes ("high" and "low") ended the list. In fact, the difference in recall of the last item between the tone and word suffix conditions was actually somewhat larger numerically for the subjects in the attend group than for subjects in the ignore group; this is clearly a finding that is inconsistent with a shortterm memory recoding account.

Descriptively, it is apparent from Figure 1 that attention instructions made a difference in the first six positions and that the suffix type (speech vs. tone) was important in the last two or three positions. This pattern corresponds to the well established dissociation of experimental influences on the early and interior positions (which come from "regular" short-term memory) from experimental influences on the last one or more positions (which come from the precategorical acoustic storage system). The focusing of precategorical acoustic storage effects upon the last position or, at most, the last few positions has recently been documented by Baddeley and Hull (1979) and by Engle (1980). If these effects were indeed restricted to the last serial position, we nevertheless would expect to see some spillover of the effects to those items occurring just before the last item, because items are probably grouped and recalled in a dependent fashion. However, the three-way interaction that would be necessary to substantiate this prediction was not reliable in the overall analysis.

Figure 1 also suggests that the attend instruction led to more errors overall than the ignore instruction. This comparison was not statistically reliable; however, it was a between-group manipulation and a larger experiment would probably provide the needed statistical evidence. Generally, any form of interference in the short-term memory system, occurring between presentation and recall, can be expected to increase errors in performance. Such effects should be nonselective across serial positions, as was the attend/ignore difference here. Crowder and Morton (1969) assigned the term "prefix effect" to such a process.

The failure to reduce recency recall selectively with the tone suffix cannot readily be interpreted as a failure to manipulate the independent variable. Since subjects in the attend group began their recall by writing down the suffix, we can be sure that attention was paid to the suffixes when they occurred. This position is reinforced by the finding that only two errors were made in suffix recall throughout the experiment. It seems that simple verbal recoding of nonverbal acoustic suffixes is not a condition sufficient to produce the interference that is characteristic of the stimulus suffix effect.

On the other hand, these results are quite consistent with the predictions of the precategorical acoustic storage model (Crowder \& Morton, 1969). According to that model, a suffix should be effective only to the extent that it contains acoustic information that is physically similar to the remaining items in the list. Since the acoustic information was identical for both the attend and ignore subjects, any suffix interference should have been equal for both groups.

\section{EXPERIMENT 2}

The results of Experiment 1 cast doubt upon interpretations that appeal to interference in short-term memory as a way of explaining the stimulus suffix effect. Recall of the last item in the memory list was not impaired by a manipulation that forced the subject to recode a nonverbal suffix into a verbal format. However, it is possible that the difficulty of the suffix recoding process was differential for the tone and word suffix conditions. If the labeling process was especially difficult for the tones, then it may have been advantageous for the subject to finish coding the list prior to coding the tone suffixes. If so, the tone suffixes might not have been expected to interfere with any prior encoding of the stimulus items. One of the purposes of Experiment 2 was to replicate Experiment 1 under conditions that were designed to reduce the plausibility of this criticism.

A second goal of Experiment 2 was to examine the role of silent articulation in this task. As indicated earlier, Spoehr and Corin (1978) were able to produce interference with a suffix that was silently mouthed by the experimenter. Although their interpretation of this phenomenon as interference in the recoding process may be incorrect, the effect itself appeared to be robust; in fact, the mouthed condition did not differ from a standard spoken suffix condition. Therefore, we sought to replicate this effect using a slightly different procedure.

Subjects in Experiment 2 received series of nine-digit lists that were presented visually rather than vocally. Subjects were instructed to read each digit aloud as it appeared in preparation for a written recall test. Having the visually presented lists read aloud provides a source of auditory information that is nearly equivalent to that which results from strictly auditory presentation (Conrad \& Hull, 1968). In addition, all lists ended with the appearance of either the word "up" or the word "down." The important manipulation was what the subjects were instructed to do with the word suf- 
fixes. Three of four groups of subjects were required to recall the particular suffix that occurred in a fashion similar to Experiment 1, by writing either " $U$ " or " $D$ " at the beginning of the recall period. One of these suffix recall groups, the "aloud" group, was required to say the suffix aloud when it appeared on the screen. A second group, the "attend" group, had merely to note the suffix in order to be able to recall it before reporting the memory items. The third recall group, the "mouthed" group, engaged in silent articulation, or mouthing, of the suffix when it occurred. Finally, a control group, the "ignore" group, was told to ignore the words when they appeared.

One important aspect of this design was that all subjects received exactly the same stimulus materials. Moveover, all three of the suffix recall groups had to recode the same suffixes into the same final format (" $U$ " or "D"). This constraint was designed to reduce the likelihood that any subsequent differences between these groups could be interpreted in terms of encoding difficulty. According to the recoding hypothesis, recall of the last few items in the list should be impaired in the three suffix recall groups relative to the control group. In contrast, the precategorical acoustic storage account predicts that recall should be impaired only in the aloud group, since only the suffix for that group contains acoustic features.

\section{Method}

Subjects. The subjects were 60 Yale University students who participated for course credit. They were assigned to the various conditions on the basis of their time of arrival in the laboratory.

Apparatus and Materials. All stimulus events were presented and controlled by a Radio Shack TRS-80 microprocessor. There were 60 experimental trials, each consisting of a random permutation of the digits 1-9. Each trial began with the word "Ready," printed in the middle of the screen, followed by the nine digits and the stimulus suffix. All stimulus events were presented in the same location of the screen and appeared in succession at a rate of approximately 2 items/sec. The suffixes, which were presented in rhythm with the digits, were the words "up" and "down." The particular suffix that occurred was determined randomly for each trial. Following the suffix, a row of asterisks appeared on the screen, signaling the beginning of the recall period. The intertrial interval was approximately $18 \mathrm{sec}$.

Procedure. All subjects were told to repeat each digit aloud as it appeared on the screen and to write their recall in order immediately after the appearance of the suffix. Subjects were instructed not to go back to make corrections or to fill in numbers out of sequence. Immediately before the 60 experimental trials, there was a short 4-trial practice session.

All subjects received exactly the same instructions, except with regard to treatment of the suffix. Subjects in the aloud group were told that when the word "up" or"down" appeared, they were to read it aloud as if it were a 10 th item. They were then to write the letter " $U$ " or " $D$," depending upon the particular suffix that occurred, in the blank to the left of the trial number as they began their recall. Subjects in the mouthed group received the same recall instructions, except that they were told to mouth the suffix in a somewhat exaggerated manner rather than say it aloud. Subjects in the attend group were told to read the suffix silently before recalling it in a manner similar to the preceding suffix recall groups. Finally, subjects in the ignore group were told not to attend to the suffix other than as a signal to begin their recall. Rather than recalling the suffix, these subjects were told to put a check mark in the space reserved for suffix recall.

In the group required to mouth the suffix item, instructions included the fact that the experimenter would be watching to see that the mouthing responses were gross enough to enable identification of the word that had been presented on that trial. The experimenter was able to make this determination easily.

\section{Results}

As in the first experiment, subjects in the suffix recall groups followed the instructions and recalled the suffixes when they occurred. Moreover, although no systematic observations were made, casual observations by the experimenter indicated that subjects treated the suffixes in a manner consistent with the respective group instructions.

The main results of interest are plotted in Figure 2, which shows the mean percentage of recall errors for each group as a function of serial position. The scoring criterion was the same as in Experiment 1: An item had to be reproduced in its correct position to be counted correct. An overall analysis of variance revealed a significant main effect of serial position $[F(8,448)=97.09$, $\mathrm{MSe}=53.02, \mathrm{p}<.01]$ and a significant interaction of group with serial position $[\mathrm{F}(24,488)=4.20$, MSe $=$ $222.88, \mathrm{p}<.01]$. The overall main effect of suffix group, however, was not significant $[F(3,56)=2.47$, $\mathrm{MSe}=1,344.59, \mathrm{p}>.05]$. Thus, as Figure 2 indicates clearly, the reliability of differences among the four groups depends on which portion of the list one looks at: Factors that affect the overall difficulty of the task are expected to elevate the obtained error rate, whereas factors acting specifically on the auditory contribution

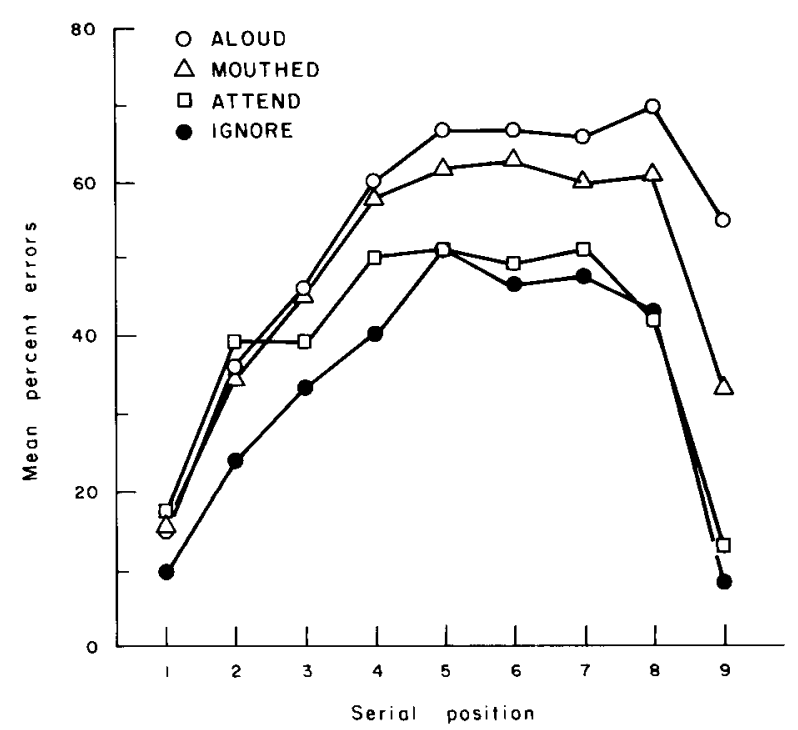

Figure 2. Serial position curves for each of the groups in Experiment 2. 
to recall should change only the recency effect. As Figure 2 shows, in the aloud and mouthed conditions, there was a consistent across-the-board performance impairment, relative to the two other groups, that can be ascribed to the difficulty of the task requirements. A similar effect occurs on the early positions for the comparison of the attend and ignore conditions. Thus, the difficulty of the task affected the subjects' overall performance on it. This commonplace observation requires no further comment before we discuss the specific decrements in performance at the terminal end of the list, for which we had reason to expect influences of the auditory system.

To assess the apparent mean differences in group recall for the last serial position, a one-way analysis of variance was performed on the normalized errors for that position. Normalized errors on the last position represent the proportion of all errors made by a subject, in a particular condition, that fell on that position. This measure can be considered a pure measure of relative recency, of how much advantage was given to the last position compared with the rest of the list. Since precategorical acoustic storage effects should be superimposed on a great many other influences on short-term memory, the normalized scores on the last position represent one of the best indications of precategorical acoustic storage working by itself. The only possible compromise to the neat separation of prerecency effects from ninth-position effects in Figure 2 is the slight separation of the aloud and mouthed groups on Positions 5-8. As we stated above, the last position could have a parasitic effect on its near neighbors if it sometimes ordinarily serves as a recall cue for them. In any case, the use of normalized last-position errors as a measure is conservative, because differences prior to the last item will reduce the magnitude of differences after normalization.

The analysis of normalized last-position errors was highly reliable $[\mathrm{F}(3,42)=28.29, \mathrm{MSe}=11.29, \mathrm{p}<.01]$. A subsequent Newman-Keuls analysis showed that all groups differed significantly from each other $(p<.01)$ except the attend and ignore groups. Thus, the statistical pattern is consistent with the picture displayed in Figure 2 . Recall of the ninth digit was impaired most by the spoken suffix in the aloud group, followed by the mouthed suffix, which produced intermediate interference, and finally, by the silently recalled and ignored suffixes, which produced no appreciable interference in the attend and ignore groups. This pattern is identical for both raw errors, as shown in Figure 2, and normalized errors.

\section{Discussion}

In many respects, the results were consistent with the results in Experiment 1. Consider first the comparison of the aloud, attend, and ignore groups. According to the recoding hypothesis, substantial and comparable interference in recall of the last few digit positions should have been produced by the suffixes in the aloud and attend groups. This is because the short-term memory processing requirements for the suffixes were identical for these two groups; the only property that distinguished the groups was that the suffixes were spoken aloud in one group and not in the other. Despite the apparent similarities, however, there were dramatic differences in recency recall for these two groups. In fact, the similar recall patterns found for the attend and ignore groups suggest that the simple requirement to process the suffix into a form suitable for immediate recall has little, if any, role in the production of the standard suffix effect.

On the other hand, the striking result of Experiment 2 was the pattern of recall of the mouthed group. There were several features of this recall pattern that were similar to the aloud group; most important, the mouthed suffix produced reliable interference in recall of the ninth item, relative to the performance of the attend and ignore groups. Thus, a suffix effect was produced without the occurrence of a suffix containing acoustic features. This finding is consistent with data reported earlier by Campbell and Dodd (1980) and Spoehr and Corin (1978). It is difficult to see how this result can be interpreted within the framework of the precategorical acoustic storage model (e.g., Crowder, 1978). However, it is equally important to emphasize that silent articulation per se cannot solely be responsible for the interference that was found in this task. If one compares the aloud and mouthed groups directly, recency recall was significantly poorer in the aloud group. Therefore, acoustic information plays a significant role in producing interference that is above and beyond the interference produced by articulation. It is noteworthy that the difference between the aloud and mouthed groups occurred primarily in the last position; these groups were nearly identical otherwise. This restriction of the precategorical acoustic storage effect to the very last part of the list is entirely consistent with the emerging generalization that was cited above (Baddeley \& Hull, 1979; Engle, 1980).

\section{GENERAL DISCUSSION}

These experiments were undertaken to investigate general short-term memory accounts of the stimulus suffix effect. In particular, we sought to test an account offered by Spoehr and Corin (1978) that placed the locus of the suffix effect at the point at which subjects recode stimulus information into a form suitable for immediate written recall. According to Spoehr and Corin, coding of the last few items in a list can be interfered with by a suffix that contains, or is recoded into, articulatory features. In Experiment 1, we attempted to produce a suffix effect with a nonverbal acoustic suffix by forcing subjects to process the suffix (a tone) verbally. Despite assurances that the subjects did indeed process 
the tone into a verbal code prior to list recall, no selective interference was obtained. In fact, subjects in this experiment processed the tone into exactly the same final recall format as they did a verbal suffix (the words "high" and "low"), yet only the verbal suffixes produced a masking effect. Moreover, in Experiment 2, subjects in the attend group processed and recalled a linguistic suffix that was presented visually, and they showed no impairment in recall of the last few items relative to a no-suffix control. It is difficult to see how any general short-term memory account of the suffix effect can accommodate these results.

These results are perhaps less surprising when one considers other findings that demonstrate the importance of acoustic information in immediate recall of final list positions. For example, it has been known for some time that lists presented vocally show a recency advantage over lists presented visually (the modality effect; Conrad \& Hull, 1968). Since it is certainly true that both visually and acoustically presented lists are fully processed in short-term memory in preparation for recall, it is unclear why the modality of presentation should be influential and, in particular, why it should be influential only on the recency portions of the list. Furthermore, as Crowder (1978) has emphasized, both the suffix and the modality effects depend identically upon the phonetic class of the stimuli that are presented. Both effects can be eliminated when consonant-vowel syllables that differ only in initial stop consonants are employed (Crowder, 1971). There is little reason to expect that conventional short-term memory mechanisms can handle such a dependency. Thus, hypotheses for the suffix effect that do not comment on the modality effect or on the nature of the phonetic segments being remembered are missing part of a large and internally consistent pattern of observations.

Nevertheless, the question remains: Why did the silent articulation (mouthed) condition in Experiment 2 produce selective interference in recall of the last item? It seems unlikely that articulatory coding in the conventional sense could be responsible for this effect. It is widely accepted that articulatory coding is a common coding format for verbal information presented visually (Hintzman, 1967). Also, we have shown that the coding of visually presented suffixes for subsequent recall is not a condition sufficient for producing a suffix effect. To resolve the question of why overtly mouthed subvocalization differs from simple silent subvocalization in its influence on the last position, it may be necessary to distinguish two levels of articulatory representation: It may be that moving the articulators themselves is different from achieving a final articulatory coding format. Articulatory coding may entail only the calling up into readiness of production routines, whereas mouthing requires their actual elicitation. As Campbell and Dodd (1980) have suggested, both the articulation process and the perception of acoustic information are temporally ordered, and it may be that such information is pro- cessed differently from information presented visually. Whatever the interpretation, it is clear that the significant interference on the last position that is produced in the mouthed group relative to the attend and ignore groups is inconsistent with the precategorical acoustic storage model as it is currently formulated.

In addition, it is equally clear that any full explanation of the suffix effect will have to include a discussion of the suffix's acoustic properties; a vocally articulated suffix produced significantly greater interference than articulation in the absence of acoustic information. In fact, the mouthed condition can now be seen as the ideal control arrangement for evaluating which portion of the suffix effect can be attributed to the auditory system. In this study, this portion resided mainly in the last item and, at most, one or two positions adjacent to the last item.

In summary, we have presented the results of two experiments that stress the importance of acoustic information in the production of the stimulus suffix effect. Both experiments provided evidence that was consistent with the precategorical acoustic storage model (Crowder \& Morton, 1969) and seemingly inconsistent with general short-term memory accounts. The picture is complicated, however, by the mouthed condition in Experiment 2. It seems that the presentation of a suffix containing acoustic properties is not always a necessary condition for the production of last-position interference that is over and above the interference that is produced elsewhere in the list. Stated differently, even the "pure" measure of precategorical acoustic storage effects has been shown here to respond to operations (e.g., mouthing) that do not affect the strictly auditory information processing machinery. It is perhaps important that mouthing recruits the gross muscular gestures that are present in lipreading (Campbell \& Dodd, 1980; Spoehr \& Corin, 1978).

\section{REFERENCES}

Baddeley, A., \& Hull, A. Prefix and suffix effects: Do they have a common basis? Journal of Verbal Learning and Verbal Behavior, 1979, 18, 129-140.

Camprell, R., \& Dodd, B. Hearing by eye. Quarterly Journal of Experimental Psychology, 1980, 32, 85-99.

ConRAD, R., \& Hull, A. Input modality and the serial position curve in short-term memory. Psychonomic Science, 1968, 10, 135-136.

Crowder, R. G. Prefix effects in immediate memory. Canadian Journal of Psychology, 1967, 21, 450-461.

Crowder, R. G. The sounds of vowels and consonants in immediate memory. Journal of Verbal Learning and Verbal Behavior, 1971, 10, 587-597.

Crowder, R. G. Mechanisms of auditory backward masking in the stimulus suffix effect. Psychological Review, 1978, 85, 502-524.

Crowder, R. G., \& Morton, J. Precategorical acoustic storage (PAS). Perception \& Psychophysics, 1969, 5, 365-373.

DALLETT, K. "Primary memory": The effects of redundancy upon digit repetition. Psychonomic Science, 1965, 3, 237-238.

EngLe, R.W. The suffix effect: How many positions are involved? Memory \& Cognition, 1980, 8, 247-252. 
Hintzman, D. L. Articulatory coding in short-term memory. Journal of Verbal Learning and Verbal Behavior, 1967, 6, 312-316.

Massaro, D. W. Perceptual images, processing time, and perceptual units in auditory perception. Psychological Review, 1972 , 79, 124-145.

Monton, J., Crowder, R. G., \& Prussin, H. A. Experiments with the stimulus suffix effect. Journal of Experimental Psychology, 1971, 91, 169-190.
Monton, J., \& Holloway, C. M. Absence of a cross-modal "suffix effect" in short-term memory. Quarterly Journal of Experimental Psychology, 1970, 22, 167-176.

Spoenr, K. T., \& Conin, W. J. The stimulus suffix effect as a memory coding phenomenon. Memory \& Cognition, 1978, 6, 583-589.

(Received for publication May 4, 1981; revision accepted March 4, 1982.) 